\title{
Warm unstable asymmetric nuclear matter: Critical properties and the density dependence of the symmetry energy
}

\author{
N. Alam, ${ }^{1,}{ }^{*}$ H. Pais,${ }^{2}$ C. Providência,${ }^{2}$ and B. K. Agrawal ${ }^{1}$ \\ ${ }^{1}$ Saha Institute of Nuclear Physics, HBNI, 1/AF Bidhannagar, Kolkata 700064, India \\ ${ }^{2}$ Department of Physics, CFisUC, University of Coimbra, 3004-516 Coimbra, Portugal
}

(Received 10 March 2017; published 23 May 2017)

\begin{abstract}
The spinodal instabilities in hot asymmetric nuclear matter and some important critical parameters derived thereof are studied by using six different families of relativistic mean-field models. The slopes of the symmetry energy coefficient vary over a wide range within each family. The critical densities and proton fractions are more sensitive to the symmetry energy slope parameter at temperatures much below its critical value $\left(T_{c} \sim\right.$ $14-16 \mathrm{MeV})$. The spread in the critical proton fraction at a given symmetry energy slope parameter is noticeably larger near $T_{c}$, indicating that the equation of state of warm asymmetric nuclear matter at subsaturation densities is not sufficiently constrained. The distillation effects are sensitive to the density dependence of the symmetry energy at low temperatures which tend to wash out with increasing temperature.
\end{abstract}

DOI: 10.1103/PhysRevC.95.055808

\section{INTRODUCTION}

Core-collapse supernovae (CCSN) [1,2] are one of the most energetic events in the Universe. Matter can reach temperatures up to $\sim 20 \mathrm{MeV}$ and the density at bounce of the collapsing core goes up to 1.5-2.0 times the nuclear saturation density. During the collapse, matter does not have enough time to reach $\beta$-equilibrium conditions [3] because the event timescale is believed to be of the order of seconds, and usually a fixed proton fraction of $y_{p} \sim 0.3[1]$ is considered for the calculation of the equation of state (EoS). The reader can refer to Ref. [4] and references therein for a recent review on the relevant thermodynamics and composition for the equation of state for CCSN, compact stars, and compact stars mergers. At densities below nuclear saturation, light [5,6] and heavy clusters [7] can form, and they can modify the neutrino transport, which will affect the cooling of the proto-neutron star [8], as neutrinos play a considerable role in the development of the shock wave during the collapse [9]. The determination of the region of densities, proton fractions, and temperatures where these instabilities exist is, therefore, very important for core-collapse simulations.

Critical properties of hot asymmetric and symmetric nuclear matter may be studied with heavy-ion collisions; in particular with nuclear reactions that involve the formation of compound nuclei or multifragmentation. These data will be important to constrain the CCSN EoS. As shown in Ref. [10], the expected range of densities and temperatures for CCSN matter just before bounce lie in the typical $(\rho, T)$ space, $\rho \sim 0.05 \rho_{0}-0.4 \rho_{0}$ and $T \sim 3-8 \mathrm{MeV}$, for nuclear multifragmentation reactions. In Ref. [11], a compilation of the critical temperatures determined from experimental data [12-15], and which generally fall above $16 \mathrm{MeV}$, is compared with theoretically determined ones from RMF models with nonlinear sigma models that have an effective mass at saturation in the range $0.58 \leqslant m^{*} / M \leqslant 0.65$, as

*naosad.alam@saha.ac.in obtained from finite nuclei spin-orbit splittings, and the incompressibility in the range $250 \leqslant K_{0} \leqslant 315 \mathrm{MeV}$, as proposed in Ref. [16]. Under these conditions, it was shown that the critical temperature from RMF models satisfies $14.2 \leqslant$ $T_{c} \leqslant 16.1 \mathrm{MeV}$, far from the value proposed in Ref. [14], where the authors have analyzed six sets of experimental data, two involving the formation of compound nuclei and four multifragmentation processes, and have determined a critical temperature of $T_{c}=17.9 \pm 0.4 \mathrm{MeV}$. To be able to reproduce the experimental critical temperature, and within RMF models that only include $\sigma$ nonlinear terms, the finite nuclei spin-orbit constraint had to be relaxed in Ref. [11] and a larger nuclear effective mass chosen. However, these experimental constraints for the critical temperature, above which matter is stable against clusterization, are for symmetric matter. Constraints for asymmetric hot matter are missing.

In Ref. [17], the authors used several methods to determine the crust-core transition, including a Thomas-Fermi calculation of the inner crust and the thermodynamical and dynamical spinodals, and they showed that, for finite temperature and fixed proton fractions (CCSN conditions), the thermodynamical method gave quite similar results to more demanding calculations, such as the Thomas-Fermi calculation.

The thermodynamical spinodal, the boundary of the instability region identified by a negative curvature free energy, is determined by equating the free-energy curvature to zero. In Ref. [18], the authors used the thermodynamical approach to analyze the liquid-gas phase transition in warm asymmetric nuclear matter, as well as stellar matter, within RMF models with and without density-dependent couplings. In particular, they discussed the isospin distillation effect; that is, the different isospin content of each phase, with the gas being more neutron rich and the liquid phase with a proton fraction close to symmetric matter. They showed that this effect is not so strong when considering models with density-dependent couplings. They calculated for each temperature the critical points of the spinodal; that is, the two points where the pressure is maximum, together with the critical temperature of the system, i.e., the temperature at which the instability region melts. The 
two models with density-dependent couplings were shown to have a region of instabilities that extended to smaller proton fractions and similar density ranges when compared with models with constant couplings, but no discussion was had on the connection of these results with the density dependence of the symmetry energy. In Ref. [19], the authors also used this method together with two others to calculate the crust-core transition and pressure at zero temperature, and using two of the families that are also going to be used in this work. They observed that this calculation gives a good estimate of the transition, as the authors of Ref. [20] also found.

In this work, the critical parameters for hot asymmetric nuclear matter for six different families of RMF models are studied by using the thermodynamical method. These six families of models have been built from three different appropriately calibrated base models. An extra term that couples the $\rho$ meson either to the $\sigma$ or $\omega$ meson is added to each of the base models to yield wide variations in the symmetry energy slope $L$. The effect of $L$ on the critical temperature, density and proton fraction is then explored. We also compare our findings with experimental results from Refs. [12-15] for the critical temperature, and the theoretical study of Ref. [11].

\section{FORMALISM}

We give a brief summary of the RMF formalism in the first section, and of the thermodynamical spinodal calculation and respective critical points in the second section.

\section{A. Extended relativistic mean-field Lagrangian}

We consider a set of families, each one characterized by the same isoscalar properties, which are described by the scalarisoscalar field $\phi$ with mass $m_{s}$, associated with the $\sigma$ meson, and the vector-isoscalar field $V^{\mu}$ with mass $m_{v}$ associated with the $\omega$ meson. The members of each family differ by their isovector properties which will be determined by the vector-isovector field $\mathbf{b}^{\mu}$ with mass $m_{\rho}$, associated with the $\rho$ meson, and the nonlinear terms that couple the $\rho$ meson to the $\sigma$ and/or the $\omega$ mesons. Nucleons, with mass $M$ and described by the spinors $\psi_{i}$, interact with and through the $\sigma, \omega$, and $\rho$ mesons, according to the Lagrangian density:

$$
\mathcal{L}=\sum_{i=p, n} \mathcal{L}_{i}+\mathcal{L}_{\sigma}+\mathcal{L}_{\omega}+\mathcal{L}_{\rho}+\mathcal{L}_{\sigma \omega \rho},
$$

where the nucleon Lagrangian reads

$$
\mathcal{L}_{i}=\bar{\psi}_{i}\left[\gamma_{\mu} i D^{\mu}-M^{*}\right] \psi_{i}
$$

with

$$
\begin{aligned}
i D^{\mu} & =i \partial^{\mu}-g_{v} V^{\mu}-\frac{g_{\rho}}{2} \boldsymbol{\tau} \cdot \mathbf{b}^{\mu}-e A^{\mu} \frac{1+\tau_{3}}{2}, \\
M^{*} & =M-g_{s} \phi .
\end{aligned}
$$

The mesonic Lagrangians are

$$
\begin{aligned}
& \mathcal{L}_{\sigma}=+\frac{1}{2}\left(\partial_{\mu} \phi \partial^{\mu} \phi-m_{s}^{2} \phi^{2}-\frac{1}{3} \kappa \phi^{3}-\frac{1}{12} \lambda \phi^{4}\right), \\
& \mathcal{L}_{\omega}=-\frac{1}{4} \Omega_{\mu \nu} \Omega^{\mu \nu}+\frac{1}{2} m_{v}^{2} V_{\mu} V^{\mu}+\frac{1}{4 !} \xi g_{v}^{4}\left(V_{\mu} V^{\mu}\right)^{2}, \\
& \mathcal{L}_{\rho}=-\frac{1}{4} \mathbf{B}_{\mu \nu} \cdot \mathbf{B}^{\mu \nu}+\frac{1}{2} m_{\rho}^{2} \mathbf{b}_{\mu} \cdot \mathbf{b}^{\mu},
\end{aligned}
$$

where $\Omega_{\mu \nu}=\partial_{\mu} V_{\nu}-\partial_{\nu} V_{\mu}, \mathbf{B}_{\mu \nu}=\partial_{\mu} \mathbf{b}_{\nu}-\partial_{\nu} \mathbf{b}_{\mu}-g_{\rho}\left(\mathbf{b}_{\mu} \times \mathbf{b}_{\nu}\right)$, and $\boldsymbol{\tau}$ are the Pauli matrices. The mesonic Lagrangian is supplemented with the following nonlinear terms that mix the $\sigma, \omega$, and $\rho$ mesons up to quartic order [21-24]:

$$
\begin{aligned}
\mathcal{L}_{\sigma \omega \rho}= & \Lambda_{1 \sigma} g_{s} g_{\rho}^{2} \phi \mathbf{b}_{\mu} \cdot \mathbf{b}^{\mu}+\Lambda_{\sigma} g_{s}^{2} g_{\rho}^{2} \phi^{2} \mathbf{b}_{\mu} \cdot \mathbf{b}^{\mu} \\
& +\Lambda_{v} g_{v}^{2} g_{\rho}^{2} \mathbf{b}_{\mu} \cdot \mathbf{b}^{\mu} V_{\mu} V^{\mu}
\end{aligned}
$$

The parameters of RMF models, which in the present case are the couplings $g_{s}, g_{v}$, and $g_{\rho}$ of the mesons to the nucleons, the nucleon bare mass $M$, the meson masses, the self-interacting coupling constants, $\kappa, \lambda$, and $\xi$, and the coupling constants of the nonlinear mixing terms, $\Lambda_{v}, \Lambda_{\sigma}$, $\Lambda_{1 \sigma}$, are fixed to nuclear properties obtained experimentally, and to astrophysical constraints [23,24].

The free-energy density is obtained from the relation

$$
\mathcal{F}=\mathcal{E}-T \mathcal{S}
$$

with the energy density $\mathcal{E}$ given by

$$
\begin{aligned}
\mathcal{E}= & \sum_{i=p, n} E_{i}+g_{v} V_{0} \rho_{v}+g_{\rho} b_{0} \rho_{3} \\
& +\frac{1}{2}\left[\left(\nabla \phi_{0}\right)^{2}+m_{s}^{2} \phi_{0}^{2}\right]+\frac{\kappa}{3 !} \phi_{0}^{3}+\frac{\lambda}{4 !} \phi_{0}^{4} \\
& -\frac{1}{2}\left[\left(\nabla V_{0}\right)^{2}+m_{v}^{2} V_{0}^{2}+\frac{\xi g_{v}^{4}}{12} V_{0}^{4}\right]-\frac{1}{2}\left[\left(\nabla b_{0}\right)^{2}+m_{\rho}^{2} b_{0}^{2}\right] \\
& -\left(\Lambda_{v} g_{v}^{2} V_{0}^{2}-\Lambda_{\sigma} g_{s}^{2} \phi^{2}-\Lambda_{1 \sigma} g_{s} \phi\right) g_{\rho}^{2} b_{0}^{2},
\end{aligned}
$$

where the energies $E_{i}$ are

$$
E_{i}=\frac{1}{\pi^{2}} \int d p p^{2} \epsilon_{i}^{*}\left(f_{i+}+f_{i-}\right), \quad i=p, n,
$$

with the equilibrium distribution functions defined as

$$
f_{i \pm}=\frac{1}{1+\exp \left[\left(\epsilon_{i}^{*} \mp v_{i}\right) / T\right]},
$$

$\epsilon_{i}^{*}=\left(p^{2}+M_{i}^{* 2}\right)^{1 / 2}, M_{i}^{*}=M-g_{s} \phi$, and the nucleon effective chemical potential

$$
v_{i}=\mu_{i}-g_{v} V_{0}-g_{\rho} t_{3 i} b_{0},
$$

where $t_{3 i}$ is the third component of the isospin operator. The entropy density $\mathcal{S}$ is calculated considering the nucleons as quasiparticles

$$
\begin{aligned}
\mathcal{S}= & -\sum_{i=n, p} \int \frac{d^{3} p}{4 \pi^{3}}\left[f_{i+} \ln f_{i+}+\left(1-f_{i+}\right) \ln \left(1-f_{i+}\right)\right. \\
& \left.+\left(f_{i+} \leftrightarrow f_{i-}\right)\right] .
\end{aligned}
$$

\section{B. Stability conditions}

In the present study, we determine the region of instability of nuclear matter constituted by protons and neutrons by calculating the spinodal surface in $\left(\rho_{p}, \rho_{n}, T\right)$ space. Stability conditions for asymmetric matter impose that the curvature matrix of the free-energy density $[18,25,26]$,

$$
\mathcal{C}_{i j}=\left(\frac{\partial^{2} \mathcal{F}}{\partial \rho_{i} \partial \rho_{j}}\right)_{T},
$$


is positive. Equation (9) can be rewritten in the form

$$
\mathcal{C}=\left(\begin{array}{ll}
\frac{\partial \mu_{n}}{\partial \rho_{n}} & \frac{\partial \mu_{n}}{\partial \rho_{p}} \\
\frac{\partial \mu_{p}}{\partial \rho_{n}} & \frac{\partial \mu_{p}}{\partial \rho_{p}}
\end{array}\right),
$$

imposing

$$
\begin{aligned}
\operatorname{Tr}(\mathcal{C}) & >0, \\
\operatorname{Det}(\mathcal{C}) & >0
\end{aligned}
$$

to fulfill the stability conditions. This is equivalent to requiring that the two eigenvalues

$$
\lambda_{ \pm}=\frac{1}{2}\left(\operatorname{Tr}(\mathcal{C}) \pm \sqrt{\operatorname{Tr}(\mathcal{C})^{2}-4 \operatorname{Det}(\mathcal{C})}\right)
$$

are positive. The largest eigenvalue is always positive and the instability region is delimited by the surface $\lambda_{-}=0$. Interesting information is given by the associated eigenvectors $\delta \rho^{ \pm}$, defined as

$$
\frac{\delta \rho^{ \pm}}{\delta \rho_{n}^{ \pm}}=\frac{\lambda^{ \pm}-\frac{\partial \mu_{n}}{\partial \rho_{n}}}{\frac{\partial \mu_{n}}{\partial \rho_{p}}} .
$$

In particular, the eigenvector associated with the eigenvalue that defines the spinodal surface determines the instability direction, i.e., the direction along which the free energy decreases. We will also calculate the critical points for each temperature $T$, which are important to define under which conditions the system is expected to be clusterized. These points satisfy simultaneously [18,27]

$$
\begin{aligned}
\operatorname{Det}(\mathcal{C}) & =0, \\
\operatorname{Det}(\mathcal{M}) & =0,
\end{aligned}
$$

with

$$
\mathcal{M}=\left(\begin{array}{ll}
\mathcal{C}_{11} & \mathcal{C}_{12} \\
\frac{\partial|\mathcal{C}|}{\partial \rho_{p}} & \frac{\partial|\mathcal{C}|}{\partial \rho_{n}}
\end{array}\right) .
$$

The thermodynamical spinodals and respective critical points will be calculated for a series of models in the next section.

\section{RESULTS}

In this section, we first briefly describe the different families of the RMF models used for the current study. Next, we present our results for the spinodal instabilities and critical points in hot asymmetric matter at different temperatures. The effect of the symmetry energy slope parameter $L$ on these quantities will be addressed as well.

\section{A. Models}

In this work, we consider six different families of RMF models; namely, NL3 $\omega \rho$ [28], TM1 $\omega \rho$ [29], $\mathrm{F}_{2 \rho}$ [24,30], NL3 $\sigma \rho$ [29], TM $1 \sigma \rho$ [29], and $\mathrm{F}_{\rho}$ [30]. The NL3 $\omega \rho$ and $\mathrm{NL} 3 \sigma \rho$ (TM $1 \omega \rho$ and TM1 $\sigma \rho$ ) families are obtained from the base model NL3 [31] (TM1 [32]). The $\mathrm{F}_{\rho}$ and $\mathrm{F}_{2 \rho}$ families are obtained from the base model BKA22 [24]. The families NL3 $\omega \rho$, TM $1 \omega \rho$, and $\mathrm{F}_{2 \rho}$ include a quartic-order

\begin{tabular}{|c|c|c|c|c|c|c|}
\hline Model & $\begin{array}{c}B / A \\
(\mathrm{MeV})\end{array}$ & $\begin{array}{c}r_{c} \\
(\mathrm{fm})\end{array}$ & $\begin{array}{c}r_{n} \\
(\mathrm{fm})\end{array}$ & $\begin{array}{l}\Delta r_{\mathrm{np}} \\
(\mathrm{fm})\end{array}$ & $\begin{array}{l}M_{\max } \\
\left(\mathrm{M}_{\odot}\right)\end{array}$ & $\begin{array}{l}R_{\max } \\
(\mathrm{km})\end{array}$ \\
\hline$F_{\rho} 1$ & -7.871 & 5.529 & 5.751 & 0.280 & 1.99 & 11.77 \\
\hline$F_{\rho} 7$ & -7.871 & 5.559 & 5.680 & 0.179 & 1.97 & 11.33 \\
\hline$F_{2 \rho} 1$ & -7.871 & 5.529 & 5.740 & 0.269 & 1.95 & 11.61 \\
\hline$F_{2 \rho} 7$ & -7.870 & 5.555 & 5.649 & 0.152 & 1.93 & 11.06 \\
\hline NL3 & -7.878 & 5.518 & 5.740 & 0.280 & 2.78 & 13.29 \\
\hline $\mathrm{NL} 3 \sigma \rho 6$ & -7.913 & 5.535 & 5.662 & 0.185 & 2.77 & 13.14 \\
\hline NL3 $3 \omega \rho 6$ & -7.921 & 5.530 & 5.667 & 0.195 & 2.76 & 12.99 \\
\hline TM1 & -7.877 & 5.541 & 5.753 & 0.270 & 2.18 & 12.49 \\
\hline TM1 $\sigma \rho 6$ & -7.923 & 5.558 & 5.686 & 0.186 & 2.15 & 12.02 \\
\hline TM1 $1 \rho 6$ & -7.791 & 5.552 & 5.689 & 0.195 & 2.13 & 11.97 \\
\hline
\end{tabular}
cross-coupling $\omega^{2} \rho^{2}$ term $\left(\Lambda_{v} \neq 0\right)$, whereas the NL3 $\sigma \rho$ and TM1 $\sigma \rho$ families have a quartic-order cross-coupling $\sigma^{2} \rho^{2}$
TABLE I. The values of the binding energy per particle $(B / A)$, charge radii $\left(r_{c}\right)$, neutron radii $\left(r_{n}\right)$, and neutron skin thickness $\left(\Delta r_{\mathrm{np}}\right)$ for the ${ }^{208} \mathrm{~Pb}$ nucleus along with the maximum mass $\left(M_{\max }\right)$ of a neutron star and corresponding radius $\left(R_{\max }\right)$ obtained for some selected models.

term $\left(\Lambda_{\sigma} \neq 0\right)$. On the other hand, a cubic-order crosscoupling $\sigma \rho^{2}$ term $\left(\Lambda_{1 \sigma} \neq 0\right)$ is included in the $\mathrm{F}_{\rho}$ family. The strengths of the cross couplings $\left(\Lambda_{v}, \Lambda_{\sigma}\right.$, and $\left.\Lambda_{1 \sigma}\right)$, and that of the coupling of the $\rho$ mesons to the nucleons $\left(g_{\rho}\right)$, are appropriately adjusted to vary the slope of symmetry energy over a wide range without compromising the properties of the finite nuclei significantly. One of the cross couplings $\Lambda_{v}$ or $\Lambda_{\sigma}$ or $\Lambda_{1 \sigma}$ is increased (decreased) and accordingly $g_{\rho}$ is also increased (decreased) in such a way that either the binding energy of ${ }^{208} \mathrm{~Pb}$ nucleus is close the experimental value or the symmetry energy at density $0.1 \mathrm{fm}^{-3}$ is exactly the same as that for the base model. Different combinations of coupling strengths yield different behavior for the density dependence of the symmetry energy. The variants of NL3 and TM1 models are obtained by varying $\Lambda_{v}$ or $\Lambda_{\sigma}$ and adjusting $g_{\rho}$ in such a way that the symmetry energy at $\rho=0.1 \mathrm{fm}^{-3}$ is equal to the one obtained for the base models $[28,33]$. The variants of BKA22 model (i.e., $\mathrm{F}_{\rho}$ and $\mathrm{F}_{2 \rho}$ families) are obtained by varying $\Lambda_{v}$ or $\Lambda_{1 \sigma}$ and adjusting $g_{\rho}$ to reproduce the binding energy of the ${ }^{208} \mathrm{~Pb}$ nucleus. All the families of models considered are consistent with the observational constraints imposed by the measured mass $\left(\sim 2 M_{\odot}\right)$ of pulsars J1614-2230 [34] and J0348+0432 [35]; see, e.g., Ref. [36] and references therein. Besides these observational constraints, there are also experimental results and first-principles calculations that can allow us to set limits on the stellar matter EoS. In Table I, we present some bulk properties of the ${ }^{208} \mathrm{~Pb}$ nucleus as well as the neutron star maximum mass and corresponding radius obtained for the models with extreme values of $L$ from each families.

In addition to these six families of models, we also consider as reference two extra models with density-dependent couplings: DD2 [6] and DDME2 [37]. In Ref. [38], it was shown that these two models satisfy a well-accepted set of laboratorial, theoretical, and observational constraints. We are, therefore, interested in comparing the behavior of these models at finite temperature with the behavior of the six families of models we are going to analyze. 

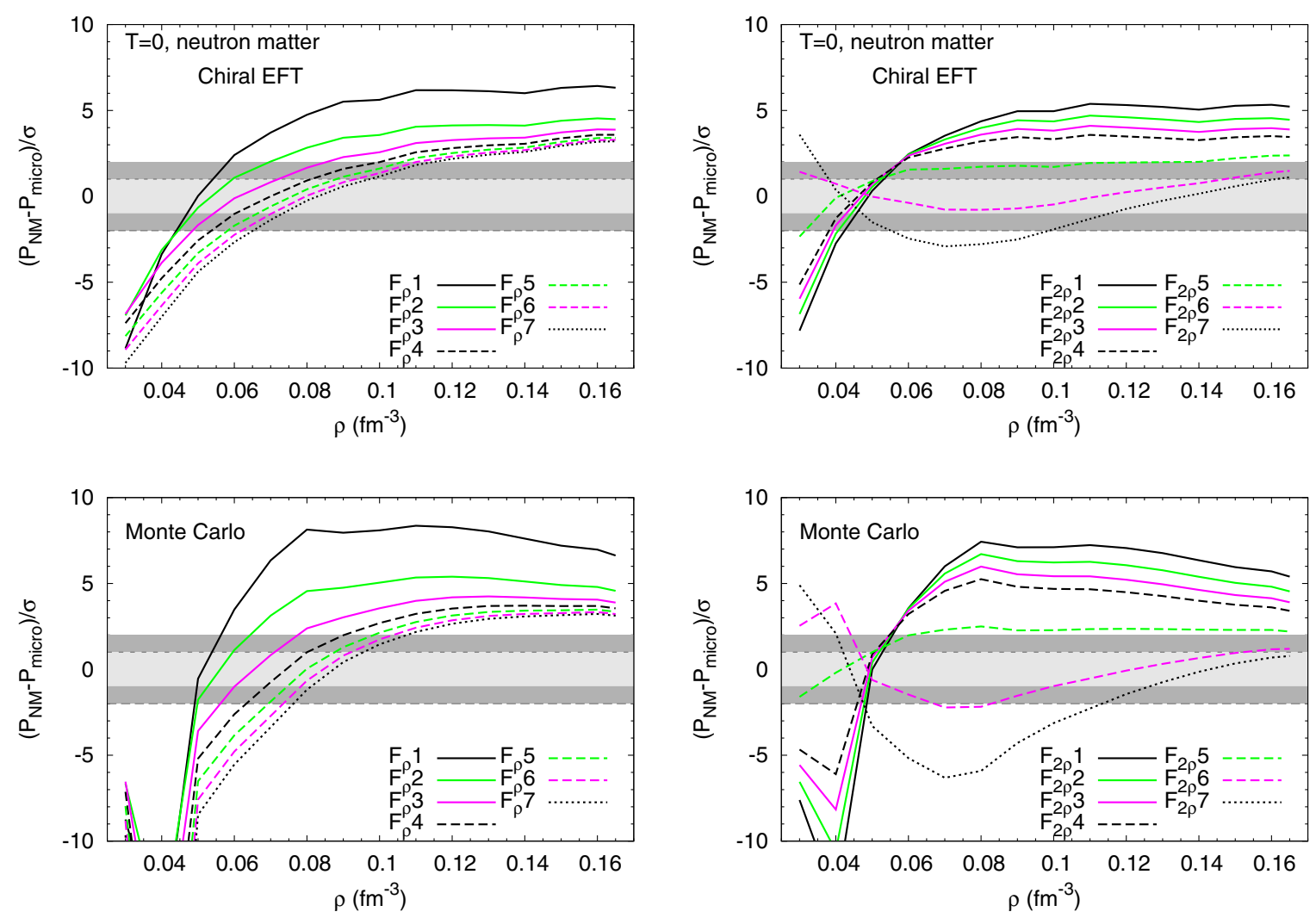

FIG. 1. Difference between the neutron matter pressure for the $\mathrm{F}_{\rho}$ (left panels) and $\mathrm{F}_{2 \rho}$ (right panels) families and the average pressure obtained from a chiral EFT [39] (top) and Monte Carlo [40] (bottom) calculations, in units of the pressure uncertainty at each density, $\sigma=\Delta P$. The gray bands represent the calculation uncertainty (light) and twice this uncertainty (dark).

In Fig. 1, we compare the neutron matter pressure of the $\mathrm{F}_{\rho}$ and $F_{2 \rho}$ families with microscopic calculations based on nuclear interactions derived from chiral effective field theory (EFT) [39] and quantum Monte Carlo techniques with realistic two- and three-nucleon interactions [40]. We show the difference from the neutron matter pressure of each model with respect to the microscopic results, normalized to the pressure uncertainty of the microscopic calculations, $\sigma=\Delta P$, at each density. These uncertainties are represented by light gray bands, and they indicate that the points that lie inside those bands are within the data limits. Also shown are dark gray bands that denote twice the calculation uncertainties, $2 \sigma$. We observe that only $\mathrm{F}_{2 \rho} 5$ and $\mathrm{F}_{2 \rho} 6$ lie in the bands' limits. All the other models fail to satisfy these constraints. Similarly, for other families, it was shown in Ref. [29] that only four models, NL3 $\omega \rho 6$, NL3 $\sigma \rho 6$, TM1 $1 \rho \rho 6$, and TM $1 \sigma \rho 6$, passed these microscopic constrains.

In Fig. 2, we show the EoSs for symmetric nuclear matter for the three base models considered together with the experimental results from collective flow data in heavy-ion collisions [41] and from the KaoS experiment [42]. The models of the NL3 family do not satisfy these constraints but the EoSs for the other models lie within the experimental bounds. However, the modelling of flow in transport simulations is a complex process and, therefore, these constraints should be considered with care. Consequently, we will also include the models of the NL3 family in the present study.
We will be discussing the effect of the density dependence of symmetry energy on the extension of the instability. To facilitate our discussions, we show in Fig. 3 the behavior of symmetry energy at subsaturation densities for the models with extreme values of the slope $L$. The models with the largest $L$ have all a very similar behavior, showing an almost linear increase of the symmetry energy with the density, typical of models that do not have nonlinear terms involving the $\rho$ meson.

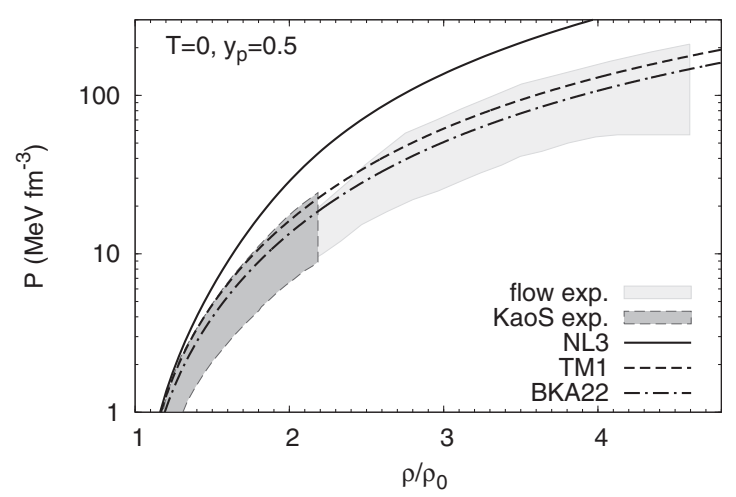

FIG. 2. Symmetric matter pressure as a function of the density for the NL3 (solid), TM1 (dashed), and BKA22 (dash-dotted) models. The colored bands are the experimental results obtained from collective flow data in heavy-ion collisions [41] (light gray) and from the KaoS experiment [42] (dark gray). 


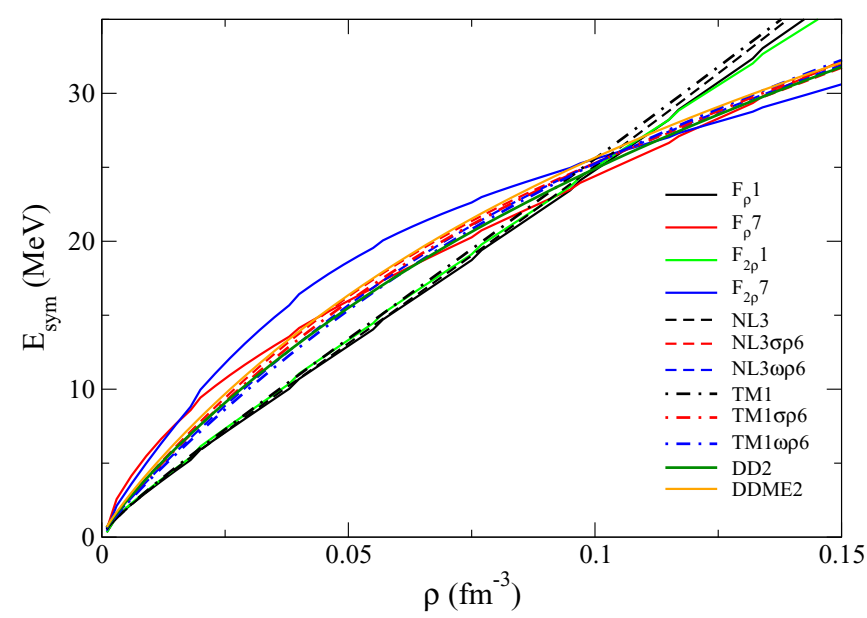

FIG. 3. Symmetry energy as a function of baryon density for the $\mathrm{F}_{\rho} 1, \mathrm{~F}_{\rho} 7, \mathrm{~F}_{2 \rho} 1, \mathrm{~F}_{2 \rho} 7$ (solid), NL3, NL3 $\sigma \rho 6$, NL3 $\omega \rho 6$ (dashed), and TM1, TM1 $\sigma \rho 6$, TM1 $\omega \rho 6$ (dash-dotted) models. The DD2 (green) and DDME2 (orange) models are also represented for comparison.

With respect to the models with the smallest $L$, the NL3 36 and TM1 $x 6$ models have a similar behavior and $L \sim 55 \mathrm{MeV}$, showing a larger symmetry energy below $\rho=0.1 \mathrm{fm}^{-3}$ than the models with large $L . F_{2 \rho} 7$ has a more extreme behavior due to its lower $L, L=45 \mathrm{MeV}$. The symmetry energy curves for the models corresponding to extreme values of $L$ cross each other at $\rho \sim 0.1 \mathrm{fm}^{-3}$, except for the $F_{\rho}$ family. The $F_{\rho} 7$ crosses $F_{\rho} 1$ at a smaller density.

\section{B. Spinodal sections and critical points}

We start with the analysis of the effects of temperature on the spinodal sections obtained for the models with extreme values of $L$, in particular the largest and the lowest of each family. In Figs. 4 and 5, we plot the spinodal sections of members 1 and 7 of the $F_{\rho}$ and $F_{2 \rho}$ families for $T=0,6$, 12, and $14 \mathrm{MeV}$, and, in Fig. 4, we also represent the line
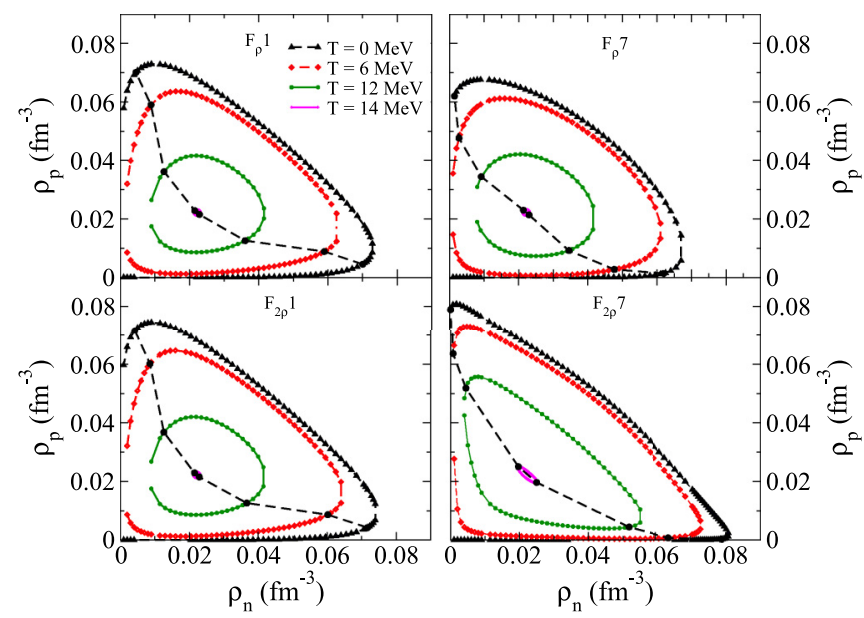

FIG. 4. Spinodal sections on the $\left(\rho_{n}, \rho_{p}\right)$ plane for $F_{\rho} 1$ (top left), $F_{\rho} 7$ (top right), $F_{2 \rho} 1$ (bottom left) and $F_{2 \rho} 7$ (bottom right) models at $T=0,6,12$, and $14 \mathrm{MeV}$.

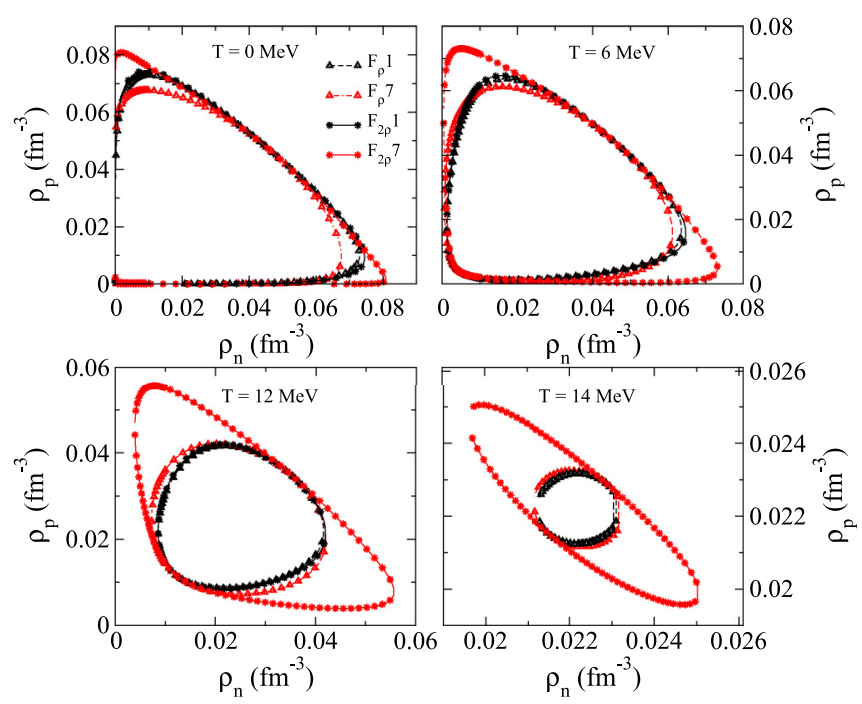

FIG. 5. Spinodal sections on the $\left(\rho_{n}, \rho_{p}\right)$ plane for $F_{\rho} 1, F_{\rho} 7$, $F_{2 \rho} 1$, and $F_{2 \rho} 7$ models at $T=0$ (top left), 6 (top right), 12 (bottom left), and 14 (bottom right) $\mathrm{MeV}$.

of critical points by a dashed line. At these points, which are common to both the binodal and the spinodal, the direction of the instability is parallel to the tangent at the spinodal, and the pressure is maximum. Some conclusions are in order: (a) The behavior with temperature is similar to the one obtained in Ref. [18], the larger the temperature the smaller the spinodal section and matter is more symmetric inside the spinodal. Eventually, at the critical temperature, the section is reduced to a point and, for larger temperatures, homogeneous matter is always stable. (b) The spinodal sections of models $F_{\rho} 1$ and $F_{2 \rho} 1$, left panels of Fig. 4, are very similar, as expected, because, these two models have very similar properties (see also Fig. 3): they are the models with the largest slope $L$ and the strength of the cross couplings is very small. (c) The same is not true for the members with the smallest values of $L, F_{\rho} 7$, and $F_{2 \rho} 7$. The spinodal of the $F_{2 \rho} 7$ model becomes larger, extending to larger asymmetries and densities. This same behavior was obtained with the NL3 and TM1 families and has been discussed in Refs. [29,43], but for dynamical spinodals. The $F_{\rho}$ family shows a different behavior, and the spinodal of the model with the smallest $L, F_{\rho} 7$, is smaller than $F_{\rho} 1$. This may be attributed to the different behavior of the symmetry energy for this model, as can be seen from Fig. 3 . In Fig. 5, where we compare the four models at different

TABLE II. Critical temperatures, and their correspondent critical densities and pressures for all the models considered in this work. The proton fraction is 0.5 .

\begin{tabular}{lccc}
\hline \hline Model & $T_{c}(\mathrm{MeV})$ & $\rho_{c}\left(\mathrm{fm}^{-3}\right)$ & $P_{c}\left(\mathrm{MeV} \mathrm{fm}^{-3}\right)$ \\
\hline DD2 & 13.73 & 0.0452 & 0.1785 \\
DD-ME2 & 13.12 & 0.0445 & 0.1556 \\
$F_{x \rho}$ & 14.01 & 0.0444 & 0.1802 \\
NL3 & 14.55 & 0.0463 & 0.1999 \\
TM1 & 15.62 & 0.0486 & 0.2365 \\
\hline \hline
\end{tabular}


temperatures, it is clear that $F_{2 \rho} 7$ is the one for which the spinodal section extends to a larger range of densities and asymmetries. This behavior is expected since this is the model with the smallest $L$.

We now consider the variations of the critical density and proton fraction with the temperature and the symmetry energy slope parameter. Before embarking on this, we would like to discuss briefly the results for the critical temperature.

The critical temperature is totally defined by the isoscalar properties of the model and, therefore, it is the same for models that only differ in the isovector properties: the critical temperatures for NL3 $x \rho, \operatorname{TM} 1 x \rho$, and $F_{x \rho}$ are the same as those for the corresponding base models NL3, TM1, and BKA22, respectively. The values of the critical temperature, density, and pressure for the base models, as well as for the DD2 and DDME2 models, are given in Table II. For the BKA22 model, the critical temperature is very close to $14 \mathrm{MeV}$, while for the TM1 model, the critical temperature is above $15 \mathrm{MeV}$, and for NL3, $T_{c}=14.55 \mathrm{MeV}$. The TM1 and $\mathrm{NL} 3 T_{c}$ values fall inside the interval of temperatures $14.2 \leqslant$ $T_{c} \leqslant 16.1 \mathrm{MeV}$, obtained in Ref. [11] from a set of RMF models with nonlinear $\sigma$ terms that have an effective mass at saturation that reproduces finite nuclei spin-orbit splittings, and an incompressibility in the range $250 \leqslant K_{0} \leqslant 315 \mathrm{MeV}$,

TABLE III. Critical densities $\rho_{c}$ and proton fractions $Y_{p c}$ for different temperatures and for all the models considered. The slope parameter $L$ and temperature $T$ are in $\mathrm{MeV}$. The critical density $\rho_{c}$ is in $\mathrm{fm}^{-3}$.

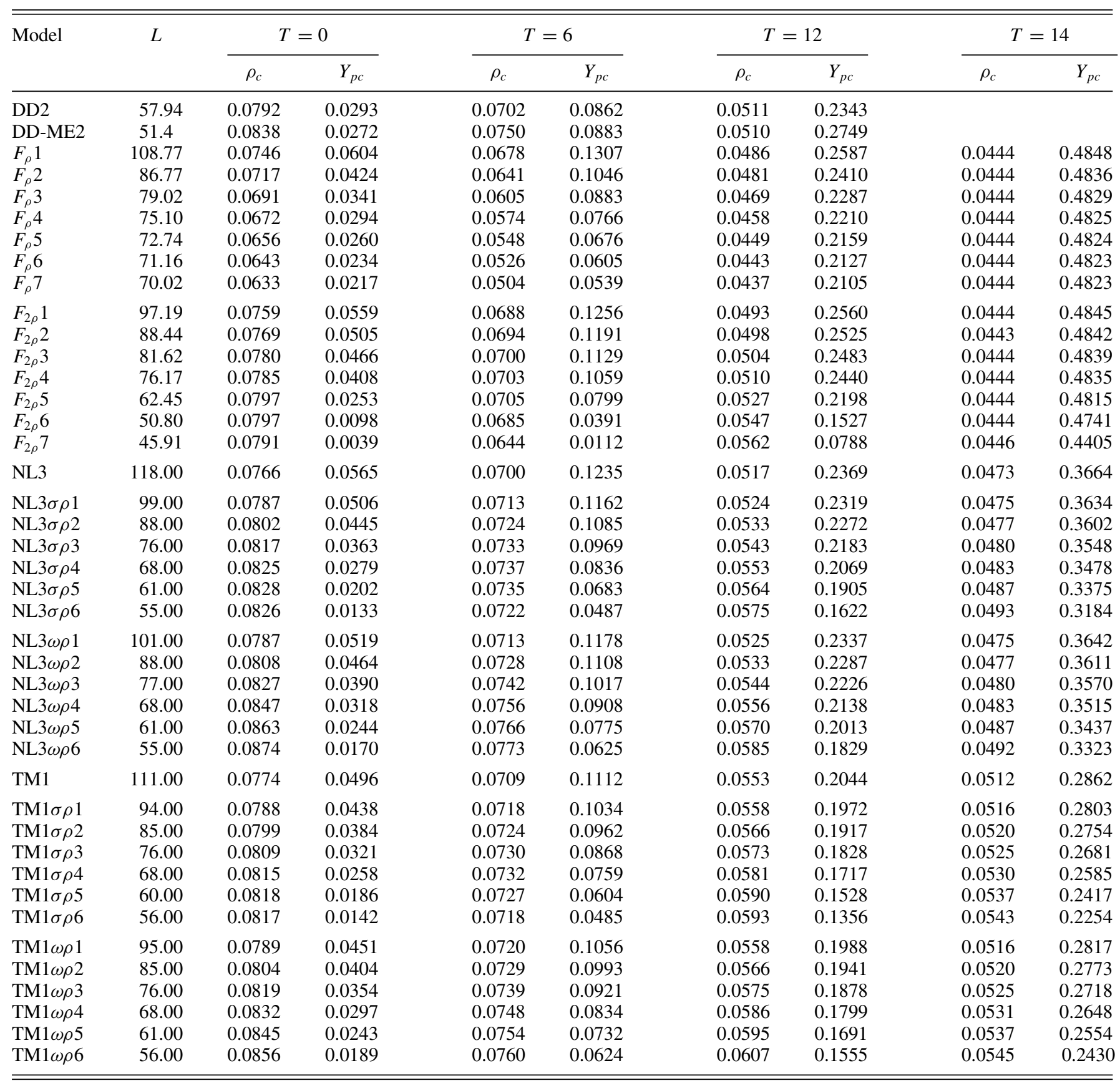



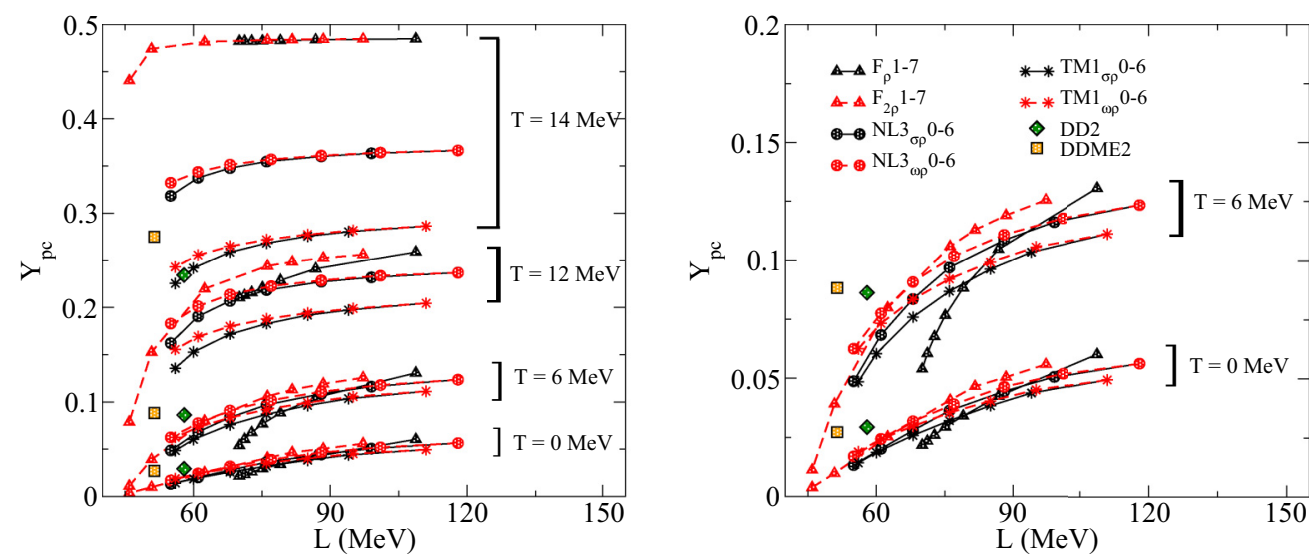

FIG. 6. Critical proton fraction, $Y_{p c}$, as a function of $L$ for several temperatures, and for all the models considered in this study. The right panel shows the results for $T=0$ and $6 \mathrm{MeV}$ only.

as proposed in Ref. [16], and the critical temperature for the BKA22 model lies very close to the bottom limit. While the incompressibilities for TM1 and NL3, 281 and $272 \mathrm{MeV}$, respectively, lie inside the range considered [11], for the BKA22 models, it is $220 \mathrm{MeV}$ and, therefore, it is outside that interval. However, the critical temperatures predicted by the models in the present study are far from the value $T_{c}=$ $17.9 \pm 0.4 \mathrm{MeV}$ obtained in Ref. [14] from the analysis of six different sets of experimental data from heavy-ion reactions. Let us stress that three of the models considered (DD2, DDME2, and NL3 $\omega \rho 6$ ) went through a set of laboratorial and theoretical constraints for neutron matter, besides predicting star masses above $2 M_{\odot}$, as identified in Ref. [38], and they predict critical temperatures below $14.55 \mathrm{MeV}$, even below $14 \mathrm{MeV}$. Besides these three models, also models NL3 $\sigma \rho 6$, TM1 $\omega \rho 6$, TM $1 \sigma \rho 6$, and $F_{2 \rho} 6$ satisfy most of these constraints: TM1 models have an incompressibility outside the range considered in Ref. [38], but well inside the range proposed in Ref. [16], and $F_{2 \rho} 6$ predicts a maximum neutron star mass just below $2 M_{\odot}$. In Ref. [14], the authors have performed a quite complete compilation of theoretical predictions for the critical temperature and, in fact, the RMF models that predict a critical temperature close to $T_{c}=17.9 \pm 0.4 \mathrm{MeV}$ do not satisfy most of the laboratorial constraints at saturation density or below. Thus, one conclusion that can be drawn is that the theoretical critical temperature predicted by the models fitted to the ground-state properties of finite nuclei and nuclear matter, and satisfying the $2 M_{\odot}$ constraint, does not agree with the experimentally extracted value of the critical temperature.

The critical points give us an indication of the phase-space region where nonhomogeneous matter is expected. The critical densities for neutron-rich matter and the respective proton fraction for $T=0,6,12$, and $14 \mathrm{MeV}$ are given in Table III, and displayed as a function of $L$ in Figs. 6 and 7. The largest temperature considered, $14 \mathrm{MeV}$, is very close to the critical temperature of the $F_{\rho}$ and $F_{2 \rho}$ models. Above the critical temperature, the models do not present instabilities and the formation of clusters is not expected. We first discuss the critical proton fraction. This quantity tells us that matter at the critical density with smaller proton fractions is stable against clusterization at the temperature considered. In the left panel of Fig. 6, the critical proton fractions are given for all the temperatures considered. This allows us to see the dependence of the critical proton fraction on the temperature. The right panel of Fig. 6 shows the same but more extensively for $T=0$ and $6 \mathrm{MeV}$. There is a clear dependence of the critical proton fraction on the slope $L$ (see right panel of Fig. 6 for more details). The critical proton fraction increases when $L$ increases: this behavior is valid for all the temperatures considered. However, it should be pointed out that, the smaller $L$, the softer is the increase of $Y_{p c}$ with temperature, for temperatures well below the critical temperature, and this

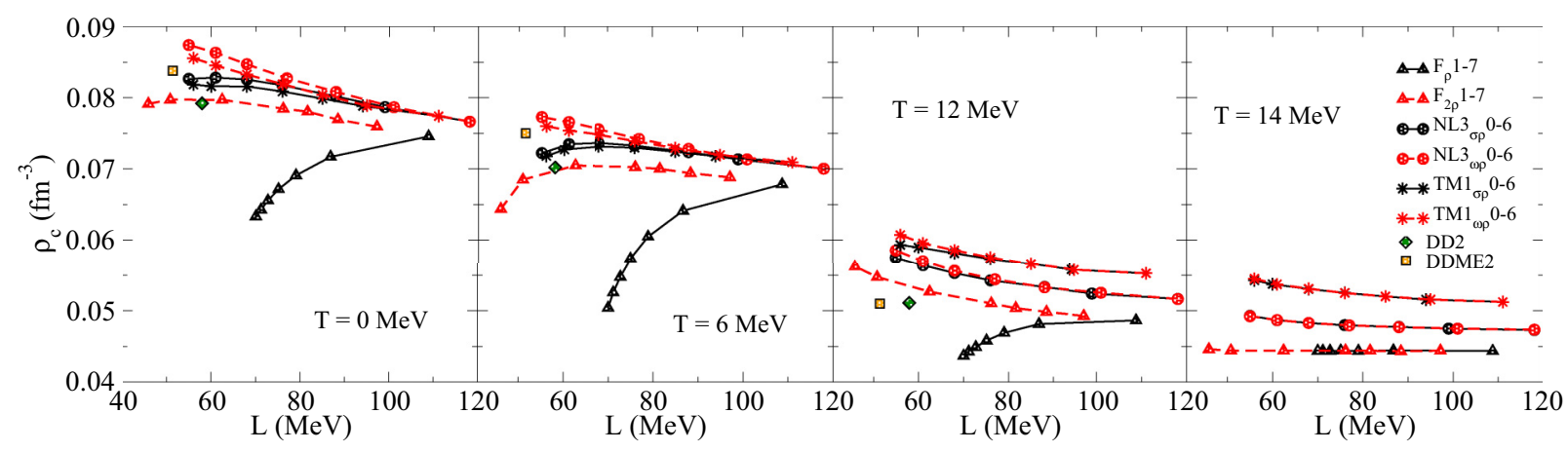

FIG. 7. Critical density $\rho_{c}$ as a function of $L$, for several temperatures, and for all the models considered in this study. 

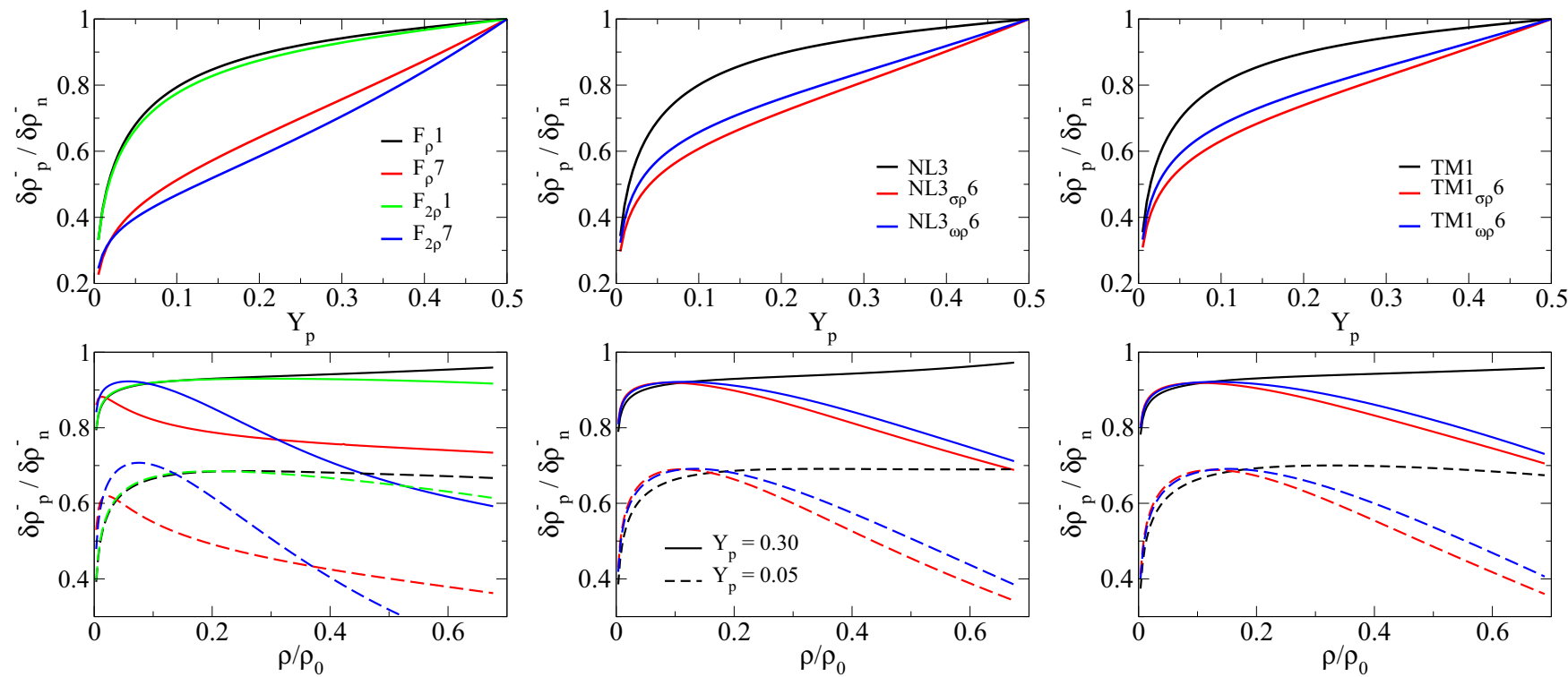

FIG. 8. The fluctuations $\delta \rho_{p}^{-} / \delta \rho_{n}^{-}$at $T=0 \mathrm{MeV}$ as a function of the proton fraction $Y_{P}$ (top panels) with $\rho=0.06 \mathrm{fm}^{-3}$, and as a function of $\rho / \rho_{0}$ (bottom panels) with $Y_{p}=0.30$ (solid) and 0.05 (dashed). The calculations shown are for the models $F_{\rho}$ and $F_{2 \rho}$ (left), $N L 3 \omega \rho$ and $N L 3 \sigma \rho$ (middle), and $T M 1 \omega \rho$ and $T M 1 \sigma \rho$ (right panels).

results in a much wider range of critical proton fractions at finite temperature than at $T=0$. For instance, the critical proton fractions for $F_{2 \rho} 7\left(F_{\rho} 1\right)$ associated with smaller (larger) values of $L$ are 0.0039 (0.0604), 0.0112 (0.1307), and $0.0788(0.2587)$ at temperature $T=0,6$, and $12 \mathrm{MeV}$, respectively.

We also notice that the spread in the values of the critical proton fraction, at a given $L$, among the various models

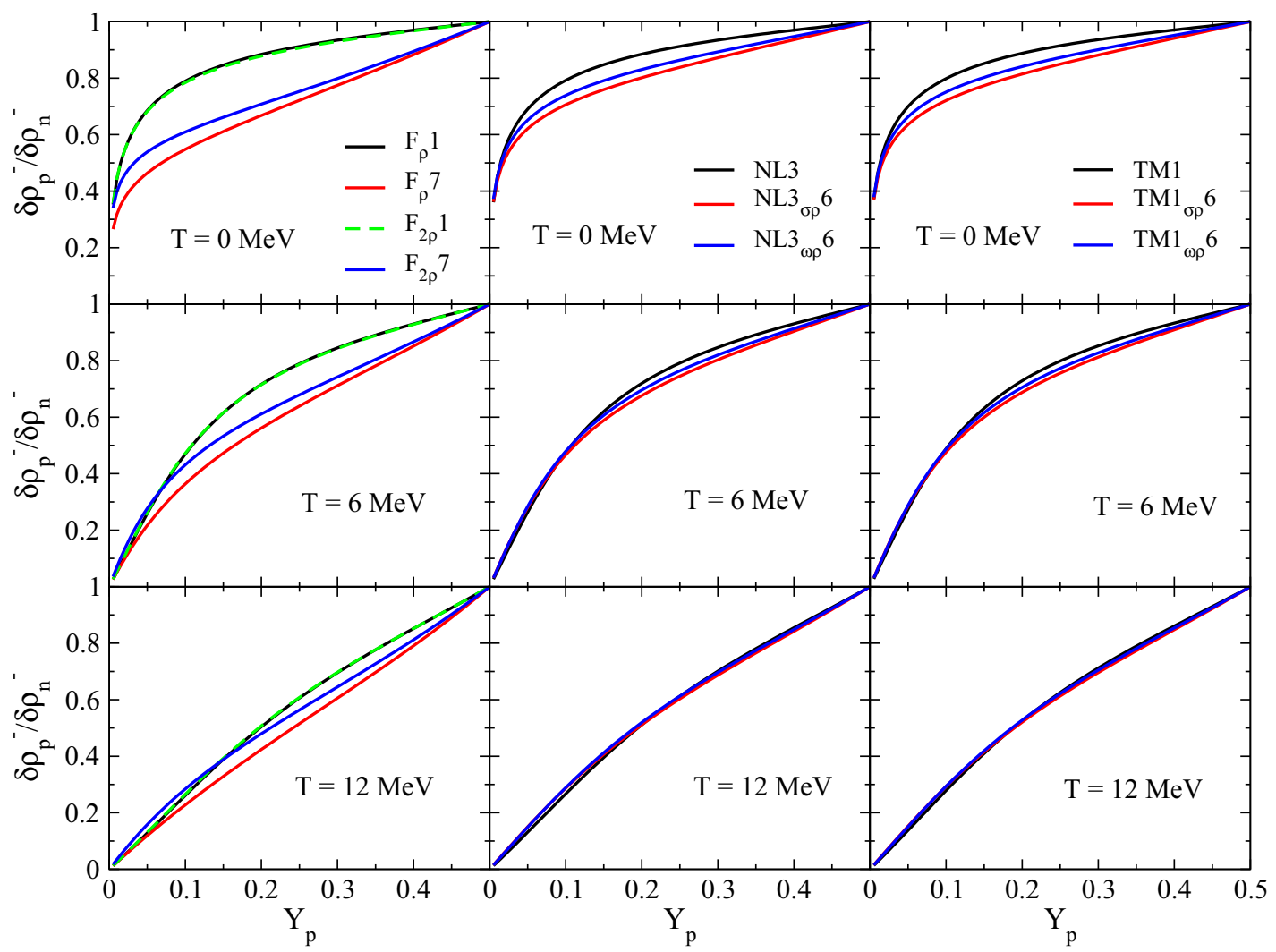

FIG. 9. The fluctuations $\delta \rho_{p}^{-} / \delta \rho_{n}^{-}$as a function of the proton fraction $Y_{P}$ for a fixed baryon density of $\rho=0.04 \mathrm{fm}^{-3}$ at $T=0 \mathrm{MeV}$ (top), $T=6 \mathrm{MeV}$ (middle), and $T=12 \mathrm{MeV}$ (bottom panels), for the $\mathrm{F}_{x \rho}$ (left), NL3 $x \rho$ (middle), and TM1 $x \rho$ (right) families. 


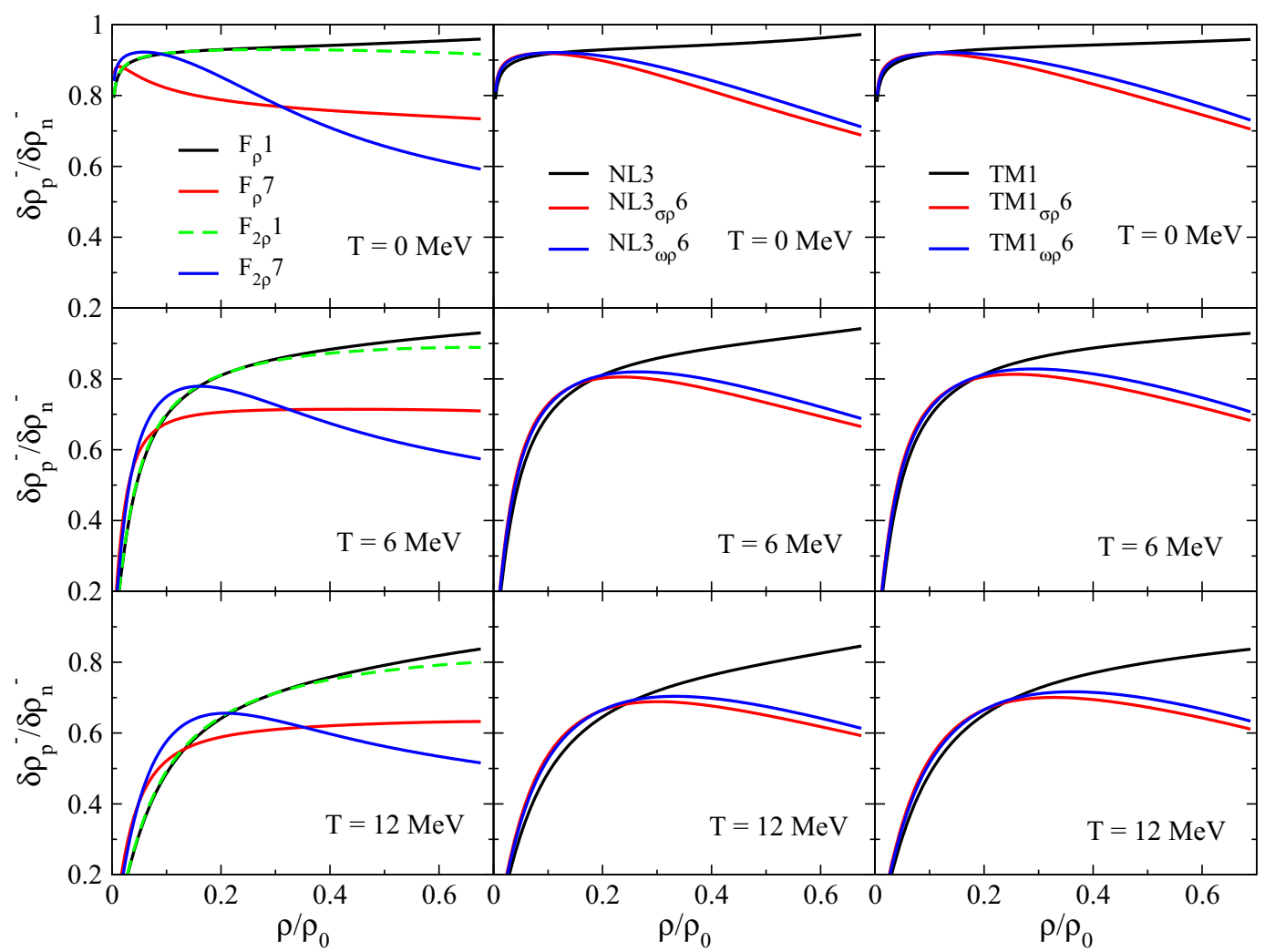

FIG. 10. The fluctuations $\delta \rho_{p}^{-} / \delta \rho_{n}^{-}$as a function of $\rho / \rho_{0}$, for a fixed proton fraction of $Y_{p}=0.3$, at $T=0 \mathrm{MeV}$ (top), $T=6 \mathrm{MeV}$ (middle),

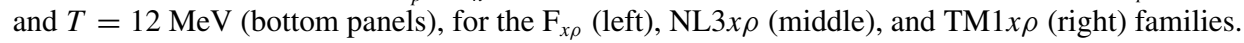

considered, increases with temperature. For a given $L$, the spread of values is not larger than $\sim 0.01$ at $T=0 \mathrm{MeV}$. At $T=6(12) \mathrm{MeV}$ the critical proton fractions spread over at least $\sim 0.03(\sim 0.1)$, for a fixed $L$. The largest temperature considered is almost coincident with, or close to, the critical temperature of the models under study. It is striking that there can be a difference of $\sim 0.25$ between the proton fractions of these models. Taking as reference $L \sim 56 \mathrm{MeV}$, a value within the constraints imposed by experiments, $Y_{p c}$ varies between 0.018 and 0.023 for $T=0 \mathrm{MeV}, 0.048$ and 0.065 for $T=6 \mathrm{MeV}, 0.136$ and 0.186 for $T=12 \mathrm{MeV}$, and between 0.225 and 0.478 for $T=14 \mathrm{MeV}$. These trends indicate that the models which are calibrated by using the bulk ground-state properties of the finite nuclei do not constrain very well the values of the critical proton fractions at finite temperatures. In fact, it should be pointed out that the large spread of the critical proton fraction close to $14 \mathrm{MeV}$ results from the fact that, for some models, BKA22 (base model for $F_{\rho}$ and $F_{2 \rho}$ families), this temperature is very close to the critical temperature, while for the TM1 models, the critical temperature is above $15 \mathrm{MeV}$, and for NL3, $T_{c}=14.55 \mathrm{MeV}$. Temperatures of the order 5-12 MeV occur in core-collapse supernova matter. We may, therefore, expect a different evolution of the supernova when different models are considered as the underlying model of the simulation. In the neutrino-trapped phase, a typical proton fraction is 0.3 , and we conclude from the left panel of Fig. 6 that, while for NL3, $F_{\rho}$, and $F_{2 \rho}$, matter at $T=14 \mathrm{MeV}$ is not clusterized, for TM1, nonhomogeneous matter still occurs under these conditions. As a reference we also include the critical proton fractions and the critical densities of the models DD2 and DDME2 in Figs. 6 and 7, since these models satisfy many well-established properties. They both have a critical temperature below $14 \mathrm{MeV}$. At $T=0$, they show a proton fraction above that predicted by the model with a symmetry energy similar to that of the six families studied. This difference grows as the temperature increases, because these models have a lower critical temperature than all the others.

Let us now discuss how the critical density $\rho_{c}$ changes with $L$ and $T$. In Fig. 7, the critical densities are plotted for the different models and temperatures considered. The model $F_{\rho}$ stands out because it is the only one that presents a critical density that increases when $L$ increases, for all temperatures. The density dependence of the symmetry energy in this model is determined by the term $\sigma \rho^{2}$, while all the others have a term $\sigma^{2} \rho^{2}$ or $\omega^{2} \rho^{2}$. Models $F_{2 \rho}$, NL $3 \sigma \rho$, and TM $1 \sigma \rho$ also show this trend for the lowest temperatures considered, 0 and $6 \mathrm{MeV}$, and $L \lesssim 60 \mathrm{MeV}$. In all other cases, $\rho_{c}$ decreases when $L$ increases. The critical densities of models DD2 and DDME2 agree with the other models. Taking again $L=56 \mathrm{MeV}$ as reference, $\rho_{c}$ decreases with $T$, from $0.080-0.087 \mathrm{fm}^{-3}$ at $T=0$, to $0.044-0.054 \mathrm{fm}^{-3}$ at $T=14 \mathrm{MeV}$, while the spread of $\rho_{c}$ increases slightly with temperature, from $0.006 \mathrm{fm}^{-3}$ at $T=0 \mathrm{MeV}$ to $0.01 \mathrm{fm}^{-3}$ at $T=14 \mathrm{MeV}$. This quantity seems, therefore, to be more constrained than the critical proton fraction. 
We address next the distillation effect referred in Refs. [18,44] within the models under discussion. This is possible from the analysis of the instability direction given by $\delta \rho_{p}^{-} / \delta \rho_{n}^{-}$. This quantity, calculated at $T=0$, has been plotted in Fig. 8 as a function of the proton fraction $Y_{P}$ for $\rho=0.06 \mathrm{fm}^{-3}$ in the top panels, and as a function of the density divided by the nuclear saturation density, $\rho / \rho_{0}$, in the bottom panels, and two different values of $Y_{p}(0.30$ and 0.05 ) for the models $F_{\rho}$ and $F_{2 \rho}$ (left), NL3 $\omega \rho$ and $\mathrm{NL} 3 \sigma \rho$ (middle), and TM1 $\omega \rho$ and TM $1 \sigma \rho$ (right panels). The two proton fractions considered are of the order of the proton fractions expected in cold catalyzed stellar matter. It is seen that the distillation effect is present in all models, the direction of instability favors a more isospin-symmetric dense matter and a more asymmetric gas phase. However, there is a clear difference between models with a large $L$ and a small $L$ : the distillation effect is much stronger for the first ones, and for a fixed proton fraction, the distillation effect increases with density, while for the second ones, after a maximum attained at $\sim 0.02 \mathrm{fm}^{-3}$, the ratio $\delta \rho_{p}^{-} / \delta \rho_{n}^{-}$decreases as the density increases. A similar behavior was obtained for density-dependent models in Ref. [18]. While $F_{2 \rho} 7$ has a behavior very similar to NL3 $x \rho 7$ and TM $1 x \rho 7$ models, with $x=\sigma$ or $\omega$, once more the $F_{\rho} 7$ shows a particular behavior, showing a smaller (larger) distillation effect for $\rho<0.04 \mathrm{fm}^{-3}$ $\left(\rho>0.04 \mathrm{fm}^{-3}\right)$ than the other models with a similar $L$. Below saturation density, models with a smaller $L$ have larger symmetry energies that disfavor a strong distillation effect.

In Figs. 9 and 10, the quantities $\delta \rho_{p}^{-} / \delta \rho_{n}^{-}$are plotted for different temperatures. We have considered the density $0.04 \mathrm{fm}^{-3}$ in the set of plots of Fig. 9 because this is the density that corresponds to clusterized matter at all temperatures. It is evident that the dependence of the distillation effects on the symmetry energy slope parameter gets washed out with the temperature, and for $T=6 \mathrm{MeV}$, the differences are already small, although there are still noticeable differences for the $F_{x \rho}$ families.

In Fig. 10, the proton fraction has been fixed to a typical value that occurs in trapped neutrino matter, $y_{p}=0.3$, and the dependence of $\delta \rho_{p}^{-} / \delta \rho_{n}^{-}$on the density is shown for different temperatures. Models of the TM1 and NL3 families are different above densities $\rho \sim 0.03-0.04 \mathrm{fm}^{-3}$, with the models with smaller slopes $L$ showing a decrease of the ratios, with a larger effect on the models with a $\sigma^{2} \rho^{2}$ nonlinear term. Models of the $F_{x \rho}$ families show larger differences at all temperatures, with the small $L$ models having larger $\delta \rho_{p}^{-} / \delta \rho_{n}^{-}$ values below $\rho \sim 0.02 \mathrm{fm}^{-3}$. The $F_{\rho} 7$ model differs again from all the other models with a similar $L$, showing a $\delta \rho_{p}^{-} / \delta \rho_{n}^{-}$ that increases monotonically with $\rho$ at finite $T$.

\section{CONCLUSION}

In the present study we analyzed the extension of the nonhomogeneous nuclear matter in the density, isospin, and temperature directions, as predicted by six different families of RMF models, together with two density-dependent models. The six families of models have been built from three different base models, whose parameters are fit to the ground-state properties of nuclei. An extra term that couples the $\rho$ meson either to the $\sigma$ or $\omega$ meson is appropriately added to each of the base models to yield the variation in the symmetry energy slope $L$ approximately between 50 and $100 \mathrm{MeV}[28,30,33]$. The thermodynamical spinodal sections are determined by the loci in phase space where the curvature matrix of the free energy is zero. These spinodal sections and lines of critical points are obtained for temperatures below the critical temperature above which there is a smooth transition from a gas to a liquid phase. The critical proton fractions and densities for a given temperature give us an indication whether clusterized matter could occur under some particular conditions. In particular, the clusterized matter is not expected at densities larger and proton fractions smaller than the corresponding critical values.

It is shown that, for a given symmetry energy slope parameter $L$, the models that include a nonlinear $\sigma-\rho$ cross coupling predict smaller critical densities and proton fractions. The effect is especially strong for the $F_{\rho}$ family, which includes a $\sigma \rho^{2}$ cross-coupling term. The critical density is more constrained. In fact, considering a slope $L=56 \mathrm{MeV}$, the spread on the critical density increases from $0.006 \mathrm{fm}^{-3}$ at $T=0$ to $\sim 0.01 \mathrm{fm}^{-3}$ at $T=14 \mathrm{MeV}$. The critical proton fraction at zero temperature increases when the slope $L$ increases and, for a given value $L$, it is almost independent of the model considered. This is not the case at finite temperature, where a spread on the proton fraction of 0.25 for $T=14 \mathrm{MeV}$ is found, when all the different models are considered. This large spread on the critical proton fraction close to $14 \mathrm{MeV}$ can be attributed to the different critical temperatures of the models under study. Since the models considered predict different critical temperatures associated with symmetric matter, the critical proton fractions at temperatures above $10 \mathrm{MeV}$ may show a large spread.

We have also analyzed the behavior of the distillation effect with temperature. In particular, previous results, concerning a smaller effect within models with a smaller slope $L$, were confirmed. Although the temperature washes out some of the differences between the models, mainly among the models of the same family, some differences remain; the stronger ones among models belonging to the $F_{x \rho}$ families.

It is observed that the $F_{\rho}$ family, which includes a cubic cross-coupling term of the type $\sigma \rho^{2}$, behaves differently as compared with the other families of models in which quartic cross-coupling terms of the type $\sigma^{2} \rho^{2}$ or $\omega^{2} \rho^{2}$ are considered. Five of six families contain at least one model that satisfies the constraints coming from microscopic calculations for pure neutron matter at subsaturation densities (see Fig. 1 and Ref. [29]); the $F_{\rho}$ family being the only one that does not satisfy this constraint.

Seemingly, these results favor the inclusion of quarticorder cross-coupling terms over the cubic-order term, although a cubic term should also be included from "naturalness" arguments [22,45]. Therefore, a more careful calibration should be undertaken, which takes into account constraints from nuclear ground-state properties, as well as constraints coming from microscopic calculations for neutron matter. 


\section{ACKNOWLEDGMENTS}

Partial support comes from Fundação para a Ciência e Tecnologia (FCT), Portugal, under Project No. UID/FIS/04564/2016 and from "NewCompStar," COST Ac- tion MP1304. H.P. is supported by FCT (Portugal) under Project No. SFRH/BPD/95566/2013. N.A. would like to thankfully acknowledge the useful discussions and the nice hospitality extended to him during his visit to University of Coimbra, when this work started.
[1] A. Mezzacappa, Annu. Rev. Nucl. Part. Sci. 55, 467 (2005).

[2] H.-T. Janka, K. Langanke, A. Marek, G. Martinez-Pinedo, and B. Mueller, Phys. Rep. 442, 38 (2007); H.-T. Janka, Annu. Rev. Nucl. Part. Sci. 62, 407 (2012); A. Burrows, Rev. Mod. Phys. 85, 245 (2013).

[3] J. R. Stone and P.-G. Reinhard, Prog. Part. Nucl. Phys. 58, 587 (2007).

[4] M. Oertel, M. Hempel, T. Klähn, and S. Typel, Rev. Mod. Phys. 89, 015007 (2017).

[5] C. J. Horowitz and A. Schwenk, Nucl. Phys. A 776, 55 (2006); S. Heckel, P. P. Schneider, and A. Sedrakian, Phys. Rev. C 80, 015805 (2009); M. Ferreira and C. Providência, ibid. 85, 055811 (2012); S. S. Avancini, C. C. Barros Jr., D. P. Menezes, and C. Providência, ibid. 82, 025808 (2010); S. S. Avancini, C. C. Barros Jr., L. Brito, S. Chiacchiera, D. P. Menezes, and C. Providência, ibid. 85, 035806 (2012).

[6] S. Typel, G. Röpke, T. Klähn, D. Blaschke, and H. H. Wolter, Phys. Rev. C 81, 015803 (2010).

[7] D. G. Ravenhall, C. J. Pethick, and J. R. Wilson, Phys. Rev. Lett. 50, 2066 (1983); C. J. Horowitz, M. A. Pérez-García, and J. Piekarewicz, Phys. Rev. C 69, 045804 (2004); T. Maruyama, T. Tatsumi, D. N. Voskresensky, T. Tanigawa, and S. Chiba, ibid. 72, 015802 (2005); G. Watanabe, T. Maruyama, K. Sato, K. Yasuoka, and T. Ebisuzaki, Phys. Rev. Lett. 94, 031101 (2005); H. Pais and J. R. Stone, ibid. 109, 151101 (2012); F. Grill, H. Pais, C. Providência, I. Vidaña, and S. S. Avancini, Phys. Rev. C 90, 045803 (2014); H. Pais, S. Chiacchiera, and C. Providência, ibid. 91, 055801 (2015).

[8] P. Haensel, Acta Phys. Pol. B 25, 373 (1994); D. Page and S. Reddy, Phys. Rev. Lett. 111, 241102 (2013).

[9] R. D. Williams and S. E. Koonin, Nucl. Phys. A 435, 844 (1985); S. Furusawa, H. Nagakura, K. Sumiyoshi, and S. Yamada, Astrophys. J. 774, 78 (2013).

[10] N. Buyukcizmeci, A. Botvina, I. Mishustin, R. Ogul, M. Hempel, J. Schaffner-Bielich, F.-K. Thielemann, S. Furusawa, K. Sumiyoshi, S. Yamada, and H. Suzuki, Nucl. Phys. A 907, 13 (2013).

[11] O. Lourenço, B. M. Santos, M. Dutra, and A. Delfino, Phys. Rev. C 94, 045207 (2016).

[12] J. B. Natowitz, K. Hagel, Y. Ma, M. Murray, L. Qin, R. Wada, and J. Wang, Phys. Rev. Lett. 89, 212701 (2002).

[13] V. A. Karnaukhov et al., Phys. Rev. C 67, 011601(R) (2003).

[14] J. B. Elliott, P. T. Lake, L. G. Moretto, and L. Phair, Phys. Rev. C 87, 054622 (2013).

[15] V. A. Karnaukhov, Phys. At. Nucl. 60, 1625 (1997); Nucl. Phys. A 734, 520 (2004); V. A. Karnaukhov et al., ibid. 780, 91 (2006); V. A. Karnaukhov, Phys. At. Nucl. 71, 2067 (2008).

[16] J. R. Stone, N. J. Stone, and S. A. Moszkowski, Phys. Rev. C 89, 044316 (2014).

[17] S. S. Avancini, S. Chiacchiera, D. P. Menezes, and C. Providência, Phys. Rev. C 82, 055807 (2010); 85, 059904(E) (2012).
[18] S. S. Avancini, L. Brito, Ph. Chomaz, D. P. Menezes, and C. Providência, Phys. Rev. C 74, 024317 (2006).

[19] H. Pais, A. Sulaksono, B. K. Agrawal, and C. Providência, Phys. Rev. C 93, 045802 (2016).

[20] C. Ducoin, J. Margueron, C. Providência, and I. Vidaña, Phys. Rev. C 83, 045810 (2011).

[21] R. J. Furnstahl, B. D. Serot, and H. B. Tang, Nucl. Phys. A 598, 539 (1996).

[22] R. J. Furnstahl, B. D. Serot, and H. B. Tang, Nucl. Phys. A 615, 441 (1997)

[23] A. Sulaksono and Kasmudin, Phys. Rev. C 80, 054317 (2009).

[24] B. K. Agrawal, Phys. Rev. C 81, 034323 (2010).

[25] V. Baran, M. Colonna, M. D. Toro, and A. B. Larionov, Nucl. Phys. A 632, 287 (1998).

[26] H. Müller and B. D. Serot, Phys. Rev. C 52, 2072 (1995).

[27] M. Modell and R. C. Reid, Thermodynamics and Its Applications, 2nd ed. (Prentice-Hall, Englewood Cliffs, 1983).

[28] J. Carriere, C. J. Horowitz, and J. Piekarewicz, Astrophys. J. 593, 463 (2003).

[29] H. Pais and C. Providência, Phys. Rev. C 94, 015808 (2016).

[30] N. Alam, A. Sulaksono, and B. K. Agrawal, Phys. Rev. C 92, 015804 (2015).

[31] G. A. Lalazissis, J. König, and P. Ring, Phys. Rev. C 55, 540 (1997)

[32] Y. Sugahara and H. Toki, Nucl. Phys. A 579, 557 (1994); K. Sumiyoshi, H. Kuwabara, and H. Toki, ibid. 581, 725 (1995).

[33] C. J. Horowitz and J. Piekarewicz, Phys. Rev. Lett. 86, 5647 (2001)

[34] P. B. Demorest, T. Pennucci, S. M. Ransom, M. S. E. Roberts, and J. W. T. Hessels, Nature (London) 467, 1081 (2010); E. Fonseca, T. T. Pennucci, J. A. Ellis, I. H. Stairs, D. J. Nice, S. M. Ransom, P. B. Demorest, Z. Arzoumanian, K. Crowter, T. Dolch, R. D. Ferdman, M. E. Gonzalez, G. Jones, M. L. Jones, M. T. Lam, L. Levin, M. A. McLaughlin, K. Stovall, J. K. Swiggum, and W. Zhu, Astrophys. J. 832, 167 (2016).

[35] J. Antoniadis, P. C. C. Freire, N. Wex, T. M. Tauris, R. S. Lynch, M. H. v. Kerkwijk, M. Kramer, C. Bassa, V. S. Dhillon, T. Driebe, J. W. T. Hessels, V. M. Kaspi, V. I. Kondratiev, N. Langer, T. R. Marsh, M. A. McLaughlin, T. T. Pennucci, S. M. Ransom, I. H. Stairs, J. v. Leeuwen, J. P. W. Verbiest, and D. G. Whelan, Science 340, 1233232 (2013).

[36] N. Alam, B. K. Agrawal, M. Fortin, H. Pais, C. Providência, A. R. Raduta, and A. Sulaksono, Phys. Rev. C 94, 052801(R) (2016).

[37] G. A. Lalazissis, T. Niksić, D. Vretenar, and P. Ring, Phys. Rev. C 71, 024312 (2005).

[38] M. Fortin, C. Providência, Ad. R. Raduta, F. Gulminelli, J. L. Zdunik, P. Haensel, and M. Bejger, Phys. Rev. C 94, 035804 (2016)

[39] K. Hebeler, J. M. Lattimer, C. J. Pethick, and A. Schwenk, Astrophys. J. 773, 11 (2013). 
[40] S. Gandolfi, J. Carlson, and S. Reddy, Phys. Rev. C 85, 032801 (2012).

[41] P. Danielewicz, R. Lacey, and W. G. Lynch, Science 298, 1592 (2002).

[42] W. G. Lynch, M. B. Tsang, Y. Zhang, P. Danielewicz, M. Famiano, Z. Li, and A. W. Steiner, Prog. Part. Nucl. Phys. 62, 427 (2009); C. Fuchs, ibid. 56, 1 (2006).
[43] H. Pais, A. Santos, L. Brito, and C. Providência, Phys. Rev. C 82, 025801 (2010).

[44] B. Jacquot, S. Ayik, Ph. Chomaz, and M. Colonna, Phys. Lett. B 383, 247 (1996).

[45] H. Müller and B. D. Serot, Nucl. Phys. A 606, 508 (1996); R. J. Furnstahl, B. D. Serot, and H.-B. Tang, ibid. 640, 505(E) (1998). 